\title{
The lectin-like domain of thrombomodulin hampers host defence in pneumococcal
} pneumonia

\author{
Marcel Schouten*,\#, J. Daan de Boer*,\#, Cornelis van 't Veer*,\#, Joris J.T.H. Roelofs", \\ Joost C.M. Meijers ${ }^{+}$, Marcel Levi ${ }^{\S}$, Edward M. Conway ${ }^{\dagger}$ and Tom van der Poll*,\#,§
}

ABSTRACT: The lectin-like domain of thrombomodulin (TM) plays an important regulatory role in sterile inflammatory conditions, but its role in severe Gram-positive infectious disease is unknown. Streptococcus pneumoniae is the most common cause of community-acquired pneumonia. The aim of this study was to determine the role of the lectin-like domain of TM in murine pneumococcal pneumonia.

Wild-type (WT) mice and mice lacking the lectin-like domain of TM (TM ${ }^{\text {LeD/LeD }}$ ) were infected intranasally with viable S. pneumoniae and either observed in a survival study or euthanised 6, 24 or $48 \mathrm{~h}$ after infection.

$T M^{\text {LeD/LeD }}$ mice had a markedly better survival in pneumococcal pneumonia when compared with WT mice. At $48 \mathrm{~h}$ post-infection with S. pneumoniae, $T \mathrm{M}^{\mathrm{LeD} / L e D}$ mice had lower bacterial loads in blood and liver, and exhibited less pulmonary inflammation, as shown by having less lung histopathology, less neutrophil influx and lower cytokine and chemokine levels. Plasma levels of pro-inflammatory cytokines were also reduced in $T M^{\mathrm{LeD} / L e D}$ mice after exposure to the infection.

Deletion of the lectin-like domain of TM improves the host defence in pneumococcal pneumonia. The lectin-like domain of TM may have a differential role in response to Grampositive or Gram-negative bacteria.

KEYWORDS: Coagulation, inflammation, sepsis, Streptococcus pneumoniae

$\mathbf{T}$ he leading cause of community-acquired pneumonia is Streptococcus pneumoniae [1]. Annually, $\sim 570000$ cases of pneumococcal pneumonia occur in the USA, accounting for $\sim 175000$ hospitalisations [2]. When associated with sepsis, mortality rates of pneumococcal pneumonia can exceed 20\% [3]. Worldwide, S. pneumoniae is responsible for an estimated 10 million deaths per year [4]. Together with the increasing incidence of antibiotic resistance to this pathogen [1], there is an urgent need to expand our knowledge of host defence mechanisms that influence the outcome of pneumococcal pneumonia and sepsis.

Thrombomodulin (TM) CD141 is a transmembrane, multidomain glycoprotein receptor that is expressed predominantly on vascular endothelial cells, but also on monocytes, neutrophils, osteoblasts, synovial cells and dendritic cells [5-7]. TM exhibits several distinct properties in coagulation, fibrinolysis, innate immunity and inflammation that are based largely on its distinct structural domains. The central domain of TM is comprised of six epidermal growth factor (EGF)-like repeats, three of which provide critical cofactor activity for the thrombin-mediated generation of activated protein $\mathrm{C}$ (APC) and of activated thrombinactivatable fibrinolysis inhibitor (TAFIa). Protein $\mathrm{C}$ activation by thrombin-TM is further augmented by the endothelial protein $C$ receptor [5-7]. APC suppresses further generation of thrombin by cleaving and inactivating coagulation cofactors Va and VIIIa. APC also has profound antiinflammatory properties [8-10]. The plasma carboxypeptidase B, TAFIa, inhibits fibrinolysis by modifying fibrinogen, rendering the fibrin resistant to plasminogen binding, and limiting plasmin generation [11]. TAFIa also inactivates the complement fragments and anaphylatoxins $\mathrm{C} 3 \mathrm{a}$ and $\mathrm{C} 5 \mathrm{a}$, thereby dampening the innate immune response [12]. In addition to its pivotal role in activating protein $\mathrm{C}$ and TAFI $[8,11]$, TM also has direct anti-inflammatory properties [5-7] that are mediated at least in part by its $\mathrm{N}$ terminal C-type lectin-like domain. This structure interferes with neutrophil adhesion, complement activation and cytokine generation [13-15], and
AFFILIATIONS

${ }^{*}$ Center for Experimental and Molecular Medicine (CEMM) Academic Medical Center, University of Amsterdam, Amsterdam, ${ }^{*}$ Center for Infection and Immunity Amsterdam (CINIMA), Academic Medical Center, University of Amsterdam, Amsterdam, "Dept of Pathology, Academic Medical Center, University of Amsterdam, Amsterdam, ${ }^{+}$Dept of Experimental Vascular Medicine, Academic Medical Center, University of Amsterdam, Amsterdam, and

${ }^{\S}$ Dept of Internal Medicine, Academic Medical Center, University of Amsterdam, Amsterdam, The Netherlands.

${ }^{f}$ Centre for Blood Research, Life Sciences Institute, Division of Hematology-Oncology, Faculty of Medicine, University of British Columbia, Vancouver, BC, Canada.

\section{CORRESPONDENCE}

M. Schouten

Center for Experimental and

Molecular Medicine (CEMM), Center for Infection and Immunity Amsterdam (CINIMA)

Academic Medical Center University of Amsterdam Meibergdreef 9 Room G2-130 1105 AZ Amsterdam The Netherlands

E-mail: m.schouten@amc.uva.nl

Received:

Jan 252012

Accepted after revision: July 102012 First published online: Aug 302012 
mice lacking the lectin-like domain of $\mathrm{TM}\left(\mathrm{TM}^{\mathrm{LeD} / \mathrm{LeD}}\right.$ mice) exhibit increased sensitivity to tissue injury in models of endotoxaemia, lung and myocardial ischaemia-reperfusion and inflammatory arthritis [13-15].

Although alterations in regulation of the coagulation system have been studied extensively in pneumonia and sepsis [8], there is limited knowledge of the role of TM in the host response to bacterial infection. As TM is highly expressed in the lung, particularly in the alveolar capillary endothelial cells $[16,17]$, we have been evaluating its participation in the response to respiratory tract infections. Mice with a point mutation in the gene encoding TM, which significantly reduces its capacity to generate APC and lowers TM antigen levels, surprisingly did not exhibit alterations in the pulmonary response to endotoxin or to respiratory pathogens, including $S$. pneumoniae [18]. Using $\mathrm{TM}^{\mathrm{LeD} / \mathrm{LeD}}$ mice and their counterpart wild-type (WT) mice, we sought to determine the in vivo role of the lectin-like domain of TM in the response to pneumococcal pneumonia.

\section{MATERIALS AND METHODS}

\section{Animals}

$\mathrm{TM}^{\mathrm{LeD} / \mathrm{LeD}}$ mice were generated as described [13] and backcrossed eight times to a C57BL/ 6 genetic background. TM $\mathrm{LeD}^{\mathrm{L} / \mathrm{Le}}$ mice express normal antigenic levels of TM, and activation of protein $C$ is intact and unaffected by the deletion of the lectinlike domain [13]. C57BL/6 WT mice were purchased from Charles River (Maastricht, the Netherlands). All experiments were approved by the Institutional Animal Care and Use Committee of the University of Amsterdam (Amsterdam, the Netherlands).

\section{Experimental infection and sampling}

Pneumonia was induced by intranasal inoculation with $\sim 5 \times 10^{4}$ CFU of $S$. pneumoniae serotype 3 (ATCC 6303; American Type Culture Collection, Rockville, MD, USA) as described $[18,19]$. Mice were observed for 1 week $(n=14$ per group), after which survivors were killed or were euthanised at predefined time-points ( $n=8$ per group per time-point). Citrated plasma was prepared from blood drawn from the inferior vena cava. Organ homogenates were prepared as described $[18,19]$. In brief, the left lung and approximately half of the spleen and liver were harvested and homogenised at $4{ }^{\circ} \mathrm{C}$ in four volumes of sterile saline using a tissue homogeniser (Biospect Products, Bartlesville, UK). Bacterial outgrowth in organ homogenates was determined as described [18, 19]. For further measurements in lung tissue, lung homogenates were diluted 1:2 with lysis buffer $(300 \mathrm{mM} \mathrm{NaCl}, 30 \mathrm{mM}$ Tris, $2 \mathrm{mM}$ $\mathrm{MgCl}_{2}, 2 \mathrm{mM} \mathrm{CaCl}$, $1 \%$ (volume/volume) Triton X-100, $\mathrm{pH}$ 7.4) with protease inhibitor mix added (4-(2-aminoethyl)benzenesulfonylfluoride (AEBSF)), EDTA-NA2, pepstatin and leupeptin, all purchased from MP Biomedical (Eindhoven, the Netherlands) and incubated for $30 \mathrm{~min}$ on ice, followed by centrifugation at $680 \times g$ for $10 \mathrm{~min}$. Supernatants were stored at $-20^{\circ} \mathrm{C}$ until analysis.

In a separate series of experiments, the trachea was exposed and canulated with a sterile 22-gauge Abbocath-T catheter (Abott, Sligo, Ireland) after which bronchoalveolar lavage (BAL) was performed by instilling and retrieving two $0.5 \mathrm{~mL}$ aliquots of saline ( $n=8$ per group per time-point). Cell counts were determined for each BAL fluid (BALF) sample in a haemocytometer (Beckman Coulter, Fullerton, CA, USA). Differential cell counts were performed on cytospins stained with Giemsa stain (Diff-Quick; Dade Behring AG, Düdingen, Switzerland).

\section{Histology and immunohistochemistry}

The right lung was fixed in $10 \%$ formalin/PBS at room temperature for $24 \mathrm{~h}$ and subsequently embedded in paraffin; $5-\mu \mathrm{m}$ thick sections were cut. Granulocyte staining was performed using fluorescein isothiocyanate-labelled antimouse-Ly-6G monoclonal antibody (Pharmingen, San Diego, CA, USA) as described [20]. Ly-6G stained slides were photographed with a microscope equipped with a digital camera (Leica CTR500; Leica Microsystems, Wetzlar, Germany). 10 random pictures were taken per slide. Stained areas were analysed with Image Pro Plus (Media Cybernetics, Bethesda, MD, USA) and expressed as a percentage of the total surface area. Slides were also stained with haematoxylin and eosin and analysed by a pathologist who was blinded to the identity of the different groups. To score lung inflammation and damage, the entire lung section was analysed with respect to the following parameters: bronchitis, interstitial inflammation, oedema, endothelialitis, pleuritis and thrombus formation. Each parameter was graded on a scale of $0-4$ (0: absent; 1 : mild; 2: moderate; 3: severe; and 4: very severe). The total histopathological score was expressed as the sum of the scores for the different parameters.

\section{Assays}

Myeloperoxidase (MPO) (HyCult Biotechnology, Uden, the Netherlands) was measured by ELISA. Tumour necrosis factor (TNF)- $\alpha$, interleukin (IL)-6, monocyte chemoattractant protein (MCP)-1, IL-12p70, interferon (IFN)- $\gamma$ and IL-10 were measured by cytometric bead array multiplex assay (BD Biosciences, San Jose, CA, USA). Keratinocyte-derived chemokine (KC) and macrophage inflammatory protein (MIP)-2 were measured by ELISA (R\&D Systems, Minneapolis, MN, USA).

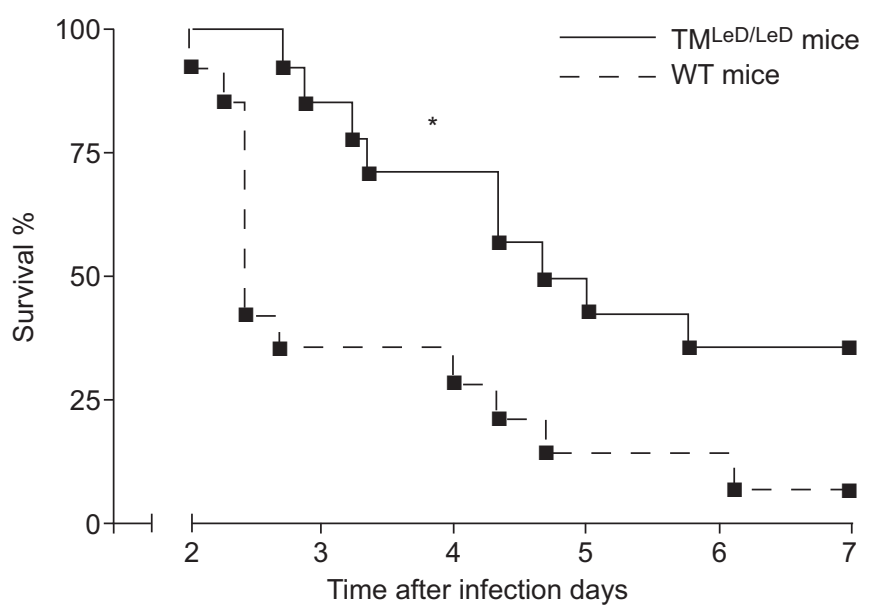

FIGURE 1. Loss of the lectin-like domain of thrombomodulin ( $\left(M^{L e D / L e D}\right.$ ) reduces mortality in murine pneumococcal pneumonia. Survival of wild-type (WT) mice and mice lacking the $T M^{L E D / L e D}$ after intranasal infection with $5 \times 10^{4}$ Streptococcus pneumoniae CFU (14 mice per group). *: $p<0.05$, statistical significance as compared with WT using log rank test. 

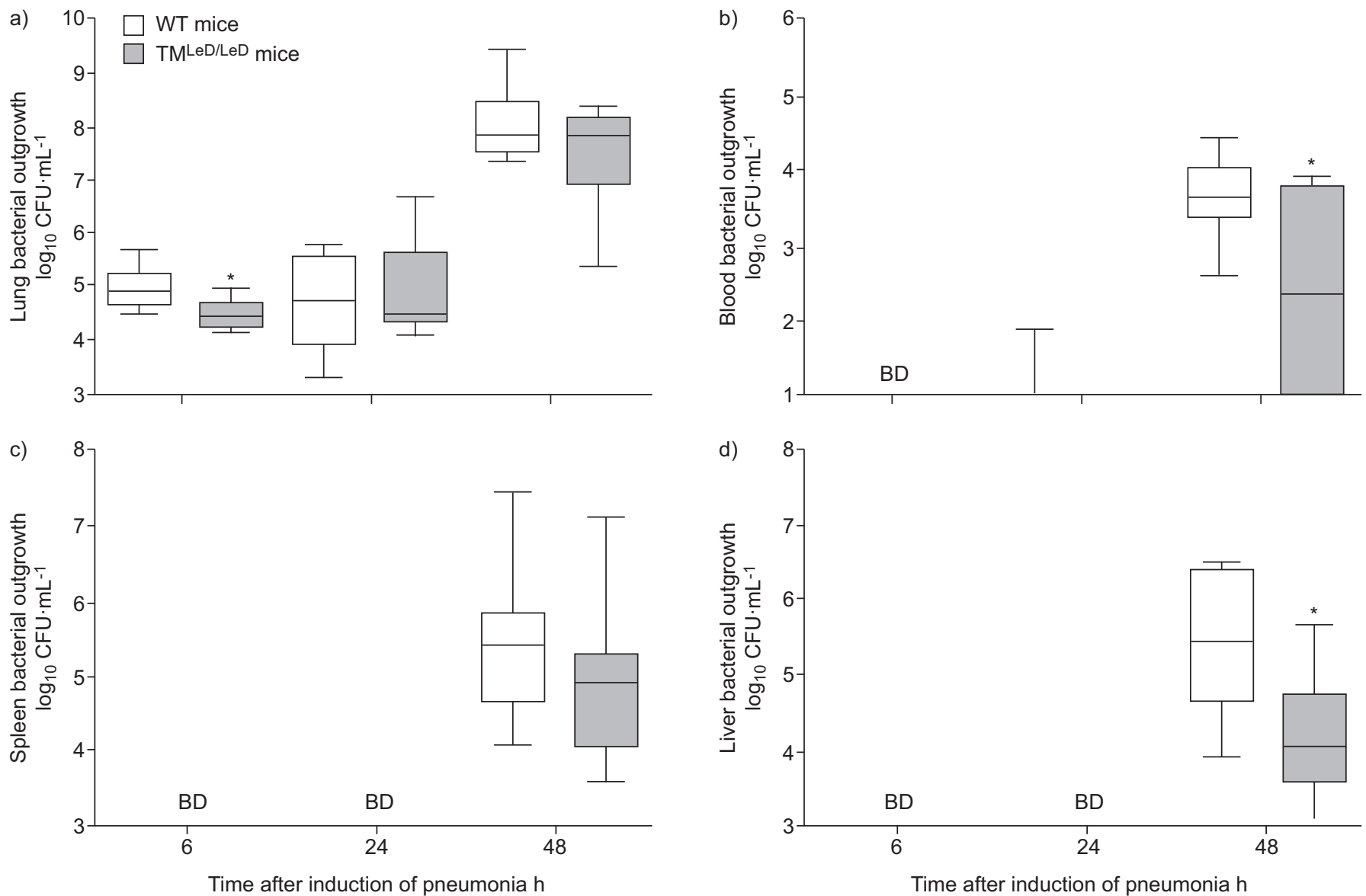

FIGURE 2. Bacterial outgrowth in blood and liver is lower in mice lacking the lectin-like domain of thrombomodulin (TM ${ }^{\text {LeD/LeD) }}$ as compared with wild-type (WT) mice after pneumococcal infection. Bacterial outgrowth in a) lung, b) blood, c) spleen and d) liver 6, 24, and $48 \mathrm{~h}$ after induction of pneumonia by intranasal inoculation with $5 \times 10^{4}$ Streptococcus pneumoniae CFU in WT and TM ${ }^{\mathrm{LeD} / \mathrm{LeD}}$ mice. Data are expressed as box-and-whisker plots depicting the smallest observation, lower quartile, median, upper quartile and largest observation (eight mice per group). BD: below detection. *: $p<0.05$, statistical significance as compared with WT using Mann-Whitney U-test.

Thrombin-antithrombin complexes (TATc) were measured by ELISA (Siemens Healthcare Diagnostics, Marburg, Germany).

\section{Statistical analysis}

Data are expressed either as box-and-whisker plots depicting the smallest observation, lower quartile, median, upper quartile and largest observation, as medians with interquartile ranges or as Kaplan-Meier plots. Differences between groups were determined with Mann-Whitney U-test or log rank test where appropriate. Analyses were performed using GraphPad Prism version 4.0 (GraphPad Software, San Diego, CA, USA). p-values of $<0.05$ were considered statistically significant.

\section{RESULTS \\ $T M^{L e D / L e D}$ mice exhibit a survival advantage after pneumococcal infection}

To determine whether the lectin-like domain of TM alters survival in response to lethal doses of S. pneumoniae, $\mathrm{TM}^{\mathrm{LeD} / \mathrm{LeD}}$ and WT mice were inoculated intranasally with the bacteria and monitored for 1 week (fig. 1 ). $\mathrm{TM}^{\mathrm{LeD} / \mathrm{LeD}}$ mice were significantly protected from the lethal infection as compared with WT mice $(\mathrm{p}<0.05)$, and started dying later than WT mice. Five out of $14 \mathrm{TM}^{\mathrm{LeD} / \mathrm{LeD}}$ mice were alive at the end of the study, whereas only one out of the 14 WT mice survived.

\section{$T M^{L e D / L e D}$ mice show diminished dissemination of the infection}

To determine whether the difference in survival between $\mathrm{TM}^{\mathrm{LeD} / \mathrm{LeD}}$ and WT mice could be attributed to a difference in antibacterial defence, we measured bacterial outgrowth 6, 24 and $48 \mathrm{~h}$ post-infection in lungs, blood and distant organs (spleen and liver) (fig. 2). At $6 \mathrm{~h}, \mathrm{TM}^{\mathrm{LeD} / \mathrm{LeD}}$ mice had slightly lower bacterial loads in their lungs than WT mice $(p<0.05)$ (fig. 2a). However, at later time-points, there were no detectable differences in lung bacterial burdens in the different genotype mice (fig. 2a). Whereas cultures in blood and distant organs were almost invariably negative during the first $24 \mathrm{~h}$ after infection (fig. $2 \mathrm{~b}-\mathrm{d}$ ), at $48 \mathrm{~h}$ the majority of mice displayed systemic infection. Notably, at this time-point, $\mathrm{TM}^{\mathrm{LeD} / \mathrm{LeD}}$ mice had significantly lower bacterial counts in blood (fig. 2b) and liver (fig. 2d) as compared with WT mice (both $\mathrm{p}<0.05$ ), whereas pneumococcal loads in spleen (fig. 2c) were not significantly different between groups.

\section{Lungs of $T M^{L E D / L e D}$ mice are relatively resistant to pro-inflammatory effects of $\mathrm{S}$. pneumoniae}

Pneumococcal pneumonia was associated with pulmonary inflammation and damage as evidenced by influx of neutrophils into the lungs and the occurrence of bronchitis, interstitial 

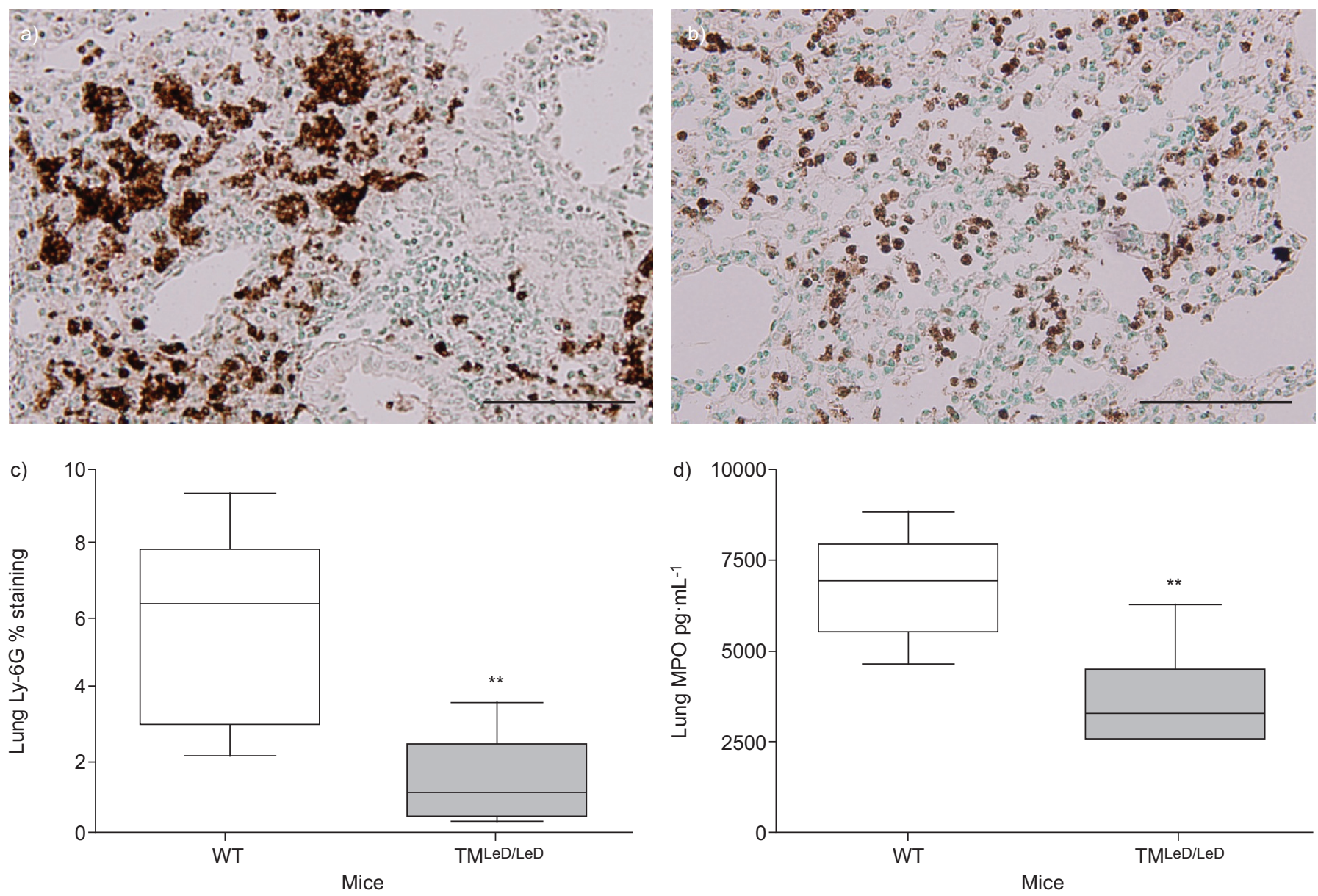

FIGURE 3. Lung neutrophil influx and myeloperoxidase (MPO) levels are lower in mice lacking the lectin-like domain of thrombomodulin (TM ${ }^{\mathrm{LeD} / \mathrm{LeD})}$ as compared with wild-type (WT) mice after pneumococcal infection. Representative slides of lung fluorescein isothyocyanate anti-mouse Ly-6G staining (brown) $48 \mathrm{~h}$ after induction of pneumonia by intranasal inoculation with $5 \times 10^{4}$ Streptococcus pneumoniae CFU in a) WT mice and b) $\mathrm{TM}^{\mathrm{LeD} / \mathrm{LeD}}$ mice. Scale bars $=100 \mu \mathrm{m}$. c) Quantitation of pulmonary Ly-6G $48 \mathrm{~h}$ after induction of pneumococcal pneumonia in WT mice and TM ${ }^{L \mathrm{LD} / L \mathrm{LD}}$ mice. d) Lung levels of MPO $48 \mathrm{~h}$ post-infection with pneumococcal pneumonia in WT mice and TM ${ }^{\mathrm{LeD} / \mathrm{LeD}}$ mice. Data are expressed as box-and-whisker plots depicting the smallest observation, lower quartile, median, upper quartile and largest observation (eight mice per group). $* *: p<0.01$, statistical significance as compared with WT using Mann-Whitney U-test.

inflammation, oedema and endothelialitis. The absence of the lectin-like domain of TM in mice results in enhanced neutrophil accumulation in the lungs subsequent to endotoxin inhalation [13]. To assess the role of the lectin-like domain of TM on neutrophil influx into the pulmonary compartment in response to pneumococcal pneumonia, we performed Ly-6G staining on lung sections and measured MPO levels in lung homogenates at 24 and $48 \mathrm{~h}$ post-infection. While there were no differences at $24 \mathrm{~h}$ (data not shown), Ly-6G positivity at $48 \mathrm{~h}$ was significantly lower in the lungs of $\mathrm{TM}^{\mathrm{LeD} / \mathrm{LeD}}$ mice than of WT mice, indicating reduced neutrophil accumulation (fig. 3a-c). MPO levels were also significantly lower in lung homogenates of $\mathrm{TM}^{\mathrm{LeD} / \mathrm{LeD}}$ mice at $48 \mathrm{~h}$ (fig. $3 \mathrm{~d}$ ). In line with these findings, mean histopathological scores of lung sections at the same time-point were lower in $\mathrm{TM}^{\mathrm{LeD} / \mathrm{LeD}}$ mice (fig. 4). To investigate whether differences in lung tissue neutrophil influx resulted in differences in cell influx in the bronchoalveolar compartment, we determined total cell counts, neutrophil numbers and MPO in BALF at 6, 24 and $48 \mathrm{~h}$ after induction of pneumococcal pneumonia. There were no differences in total cell counts and neutrophil numbers between $\mathrm{TM}^{\mathrm{LeD} / \mathrm{LeD}}$ and WT mice at any of the time-points studied (table 1). Moreover, BALF MPO levels were not different (not shown).

We next quantified the levels of several chemokines and cytokines in lung homogenates at 6, 24 and $48 \mathrm{~h}$ post-infection (table 2). At 6 and $24 \mathrm{~h}$, no differences in lung chemokines and cytokines were detected between the groups. However, at $48 \mathrm{~h}$, lung homogenates from $\mathrm{TM}^{\mathrm{LeD} / \mathrm{LeD}}$ mice had significantly lower levels of the chemokines KC and MIP-2 $(p<0.05$ and $p<0.01$ respectively), and reduced levels of TNF- $\alpha(p<0.001)$, IL-6, IL-10, IFN- $\gamma$ and MCP-1 (p<0.05) (table 2).

\section{Reduced plasma cytokine levels in $T M^{L e D / L e D}$ mice after S. pneumoniae infection}

To further investigate the role of the lectin-like domain of TM in response to pneumococcal pneumonia we measured inflammatory markers in plasma. The differential inflammatory response between $\mathrm{TM}^{\mathrm{LeD} / \mathrm{LeD}}$ mice and WT mice was already evident in plasma at $24 \mathrm{~h}$ post-infection, at which point levels of TNF- $\alpha$, IL- 6 and MCP-1 were significantly lower in 

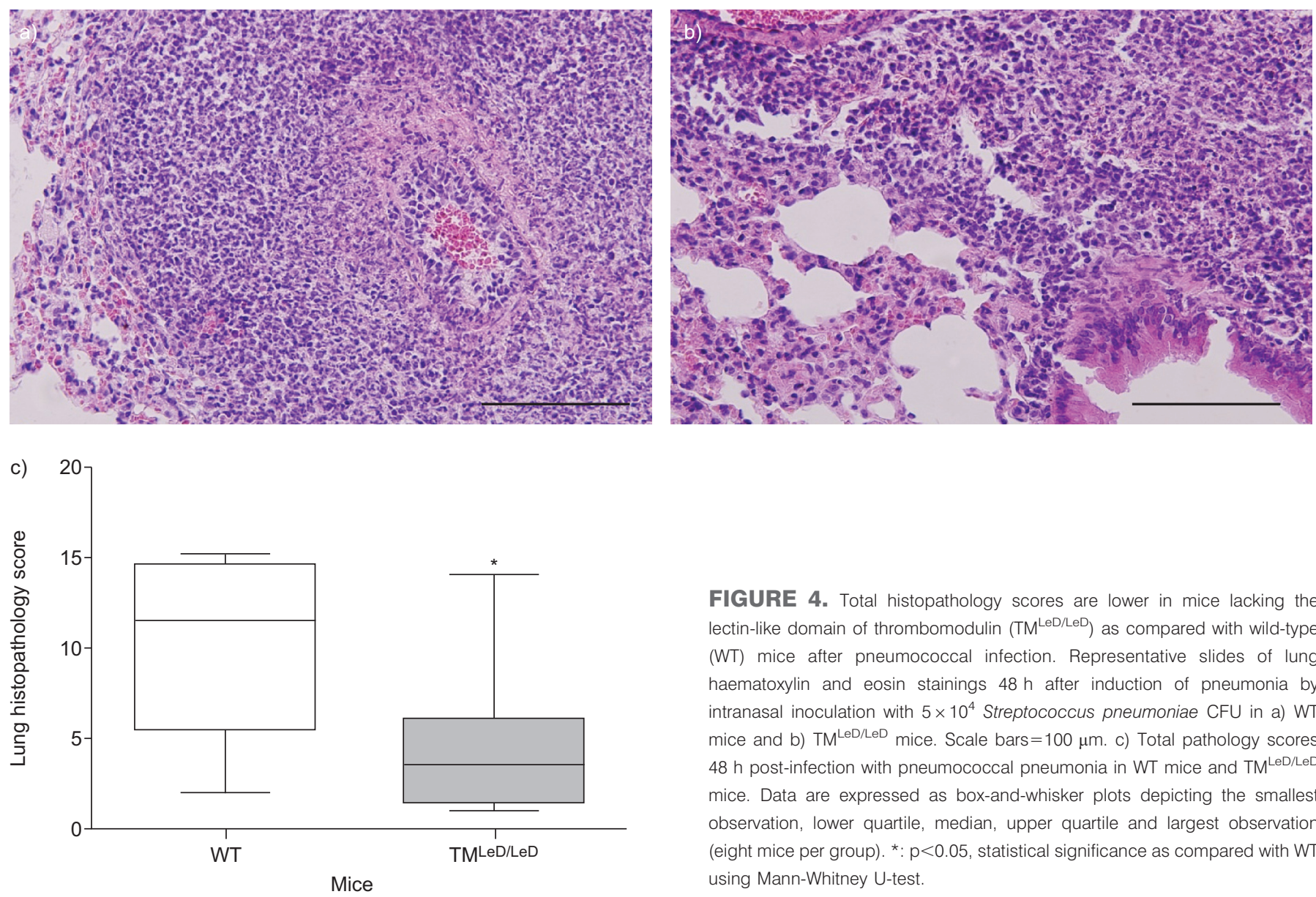

FIGURE 4. Total histopathology scores are lower in mice lacking the lectin-like domain of thrombomodulin ( $\left.\mathrm{TM}^{\mathrm{LeD} / \mathrm{LeD}}\right)$ as compared with wild-type (WT) mice after pneumococcal infection. Representative slides of lung haematoxylin and eosin stainings $48 \mathrm{~h}$ after induction of pneumonia by intranasal inoculation with $5 \times 10^{4}$ Streptococcus pneumoniae CFU in a) WT mice and b) $T M^{\text {LeD/LeD }}$ mice. Scale bars $=100 \mu \mathrm{m}$. c) Total pathology scores $48 \mathrm{~h}$ post-infection with pneumococcal pneumonia in WT mice and TM ${ }^{\text {LeD/LeD }}$ mice. Data are expressed as box-and-whisker plots depicting the smallest observation, lower quartile, median, upper quartile and largest observation (eight mice per group). *: $p<0.05$, statistical significance as compared with WT using Mann-Whitney U-test.

$\mathrm{TM}^{\mathrm{LeD} / \mathrm{LeD}}$ mice than in WT mice (table 3), a difference that was sustained until 48 h. Plasma levels of IL-10, IL-12 and IFN- $\gamma$ were below the detection limit of the assays (not shown).

\section{$T M^{L e D / L e D}$ mice exhibit a dampened coagulation response to S. pneumoniae}

We also evaluated whether the lectin-like domain of TM modified pneumococcal pneumonia-induced changes in coagulation activation by measuring TATc levels in lung homogenates and plasma. No differences in TATc levels were noted either at 6 and $24 \mathrm{~h}$ post-infection in plasma or in lung homogenates. However, at $48 \mathrm{~h}$, when plasma TATc levels remained similar in both groups of mice (data not shown), TATc levels were significantly lower in lung tissue from $\mathrm{TM}^{\mathrm{LeD} / \mathrm{LeD}}$ mice as compared with WT mice $(\mathrm{p}<0.05)$ (fig. 5).

\section{DISCUSSION}

Coagulation and inflammation are highly integrated and interactive participants in the immediate host response to infection. TM occupies a central role in the regulation of coagulation and inflammation, with responsibility for its specific functions relying on the integrity of its distinct structural domains. Thus, the EGF-like repeats provide cofactor activity for thrombin-mediated activation of protein $\mathrm{C}$ and TAFI, resulting in dampening of coagulation, fibrinolysis and complement, while the $\mathrm{N}$-terminal lectin-like domain exhibits a number of anti-inflammatory properties [5-7]. Using mouse models to

study the host response to bacterial pneumonia, we have previously investigated the role of the EGF-like repeat of TM, which is responsible for protein $\mathrm{C}$ activation. $\mathrm{TM}^{\text {Pro/Pro }}$ mice, which have moderately diminished levels of TM antigen and a dramatic reduction in its capacity to support thrombin-mediated activation of protein $\mathrm{C}$, did not exhibit TM-dependent changes in the pro-coagulant or inflammatory response to bacterial pneumonia as compared with WT mice [18]. Here, we similarly studied the role of the lectin-like domain of TM in a mouse model of community-acquired pneumonia caused by $S$. pneumoniae. This domain of TM does not participate in the generation of APC. In contrast to sterile inflammation models, in which loss of the lectin-like domain of TM renders mice more sensitive to lung and myocardial ischaemia-reperfusion injury, inflammatory arthritis and endotoxaemia [13-15], loss of the lectin-like domain of TM appears to protect the host in response to pneumococcal pneumonia, as reflected by improved survival, reduced bacterial growth and dissemination, and attenuated inflammatory and pro-coagulant response in $\mathrm{TM}^{\mathrm{LeD} / \mathrm{LeD}}$ mice as compared with WT mice.

This is the first report in which viable Gram-positive bacteria were used to evaluate the in vivo role of the lectin-like domain of TM. In our study, pneumococcal loads were transiently lower in the lungs of $\mathrm{TM}^{\mathrm{LeD} / \mathrm{LeD}}$ mice $6 \mathrm{~h}$ post-infection. This difference could not be attributed to altered inflammatory cell numbers, as BALF total cell counts and neutrophil numbers 


\begin{tabular}{|c|c|c|c|c|c|c|}
\hline & \multicolumn{2}{|c|}{$6 \mathrm{~h}$ post-infection } & \multicolumn{2}{|c|}{$24 \mathrm{~h}$ post-infection } & \multicolumn{2}{|c|}{$48 \mathrm{~h}$ post-infection } \\
\hline & WT & $T M^{\text {LeD/LeD }}$ & WT & TM $M^{\text {LeD/LeD }}$ & WT & TM LeD/LeD \\
\hline Total cell counts $10^{6}$ per $\mathrm{mL}^{-1}$ & $3.69(2.24-6.53)$ & $3.19(2.88-3.60)$ & $2.87(2.44-5.45)$ & $4.18(2.90-5.20)$ & $3.80(2.65-8.28)$ & $4.03(3.74-10.6)$ \\
\hline
\end{tabular}

were not different between $\mathrm{WT}$ and $\mathrm{TM}^{\mathrm{LeD} / \mathrm{LeD}}$ mice at this early time-point after infection. Notably, noninfected $\mathrm{TM}^{\mathrm{LeD} / \mathrm{LeD}}$ mice display a clearly enhanced neutrophil accumulation in the lung interstitium in peribronchial locations [13], which is expected to facilitate the immediate antibacterial response upon infection of the lower airways [21]. However, the difference became more prominent at $48 \mathrm{~h}$ post-infection, when bacterial loads in the blood and liver were found to be markedly lower in $\mathrm{TM}^{\mathrm{LeD} / \mathrm{LeD}}$ than in WT mice. These data suggest that killing of S. pneumoniae at the lung-blood interface, i.e. at the endothelial cell layer or in the systemic compartment itself, is enhanced in the absence of the lectin-like domain of TM. It is possible that this may be due to excess complement activation at that site due to loss of the lectin-like domain of TM that otherwise downregulates complement activation [22]. Indeed, $\mathrm{TM}^{\mathrm{LeD} / \mathrm{LeD}}$ mice have lower basal plasma C3 levels and a reduced $\mathrm{CH} 50$ (the amount of serum that causes $50 \%$ haemolysis of antibody-sensitised sheep erythrocytes), both of which are indicative of excess complement activation [15]. Considering the important role that complement activation plays in host defence against pneumococci in vivo [23], it is conceivable that the protective phenotype of $\mathrm{TM} \mathrm{LeD}^{\mathrm{LeD}}$ mice is at least in part explained by increased clearance of pneumococci from the bloodstream via augmented activation of the complement system.

In our study, $\mathrm{TM}^{\mathrm{LeD} / \mathrm{LeD}}$ mice displayed less evidence of local and systemic inflammation in response to $S$. pneumoniae infection. This was reflected by lower plasma levels of TNF- $\alpha$, IL-6 and MCP-1, less histological evidence of lung damage, lower levels of cytokines and chemokines in lung homogenates, fewer neutrophils in the infected lungs, reduced secondary activation of coagulation as measured by TATc, and finally by relative resistance to lethal doses of the bacteria. Although appearing to be contradictory to previous reports in which $\mathrm{TM}^{\mathrm{LeD} / \mathrm{LeD}}$ mice exhibited a heightened inflammatory response, those studies were performed with endotoxin or nonbacterial stresses, rather than with live Gram-positive bacteria [13]. Indeed, the current findings suggest that TM via its lectin-like domain may mediate a differential host response to Gram-negative versus Gram-positive bacteria. The lectin-like domain of TM binds to the carbohydrate Lewis $\mathrm{Y}$ antigen of Gram-negative bacteria and induces agglutination and opsonisation of Gram-negative organisms, including

TABLE 2 Pulmonary cytokine and chemokine levels in wild-type (WT) mice and mice lacking the lectin-like domain of thrombomodulin (TM $\left.{ }^{\mathrm{LeD} / \mathrm{LeD}}\right)$ at 6,24 and $48 \mathrm{~h}$ post-infection with pneumococcal pneumonia

\begin{tabular}{|c|c|c|c|c|c|c|}
\hline & \multicolumn{2}{|c|}{$6 \mathrm{~h}$ post-infection } & \multicolumn{2}{|c|}{$24 \mathrm{~h}$ post-infection } & \multicolumn{2}{|c|}{$48 \mathrm{~h}$ post-infection } \\
\hline MIP-2 $\mathrm{ng} \cdot \mathrm{mL}^{-1}$ & ND & ND & $3.66(3.16-4.52)$ & $3.38(2.99-3.81)$ & $11.8(6.65-16.6)$ & $2.77(2.23-5.54)^{\star *}$ \\
\hline TNF- $\alpha \mathbf{p g} \cdot \mathrm{mL}^{-1}$ & $20.2(12.7-26.0)$ & $23.1(15.5-30.1)$ & $5.17(2.50-6.31)$ & $2.50(2.50-13.1)$ & $116(83.9-150)$ & $8.10(4.24-39.7)^{*}$ \\
\hline $\mathrm{IL}-6 \mathrm{pg} \cdot \mathrm{mL}^{-1}$ & $36.4(25.4-41.5)$ & $44.8(30.5-67.2)$ & $19.1(5.40-123)$ & $7.63(5.38-18.3)$ & $889(501-1174)$ & $58.8(17.0-594)^{\star * *}$ \\
\hline $\mathrm{IL}-12 \mathrm{pg} \cdot \mathrm{mL}^{-1}$ & $\mathrm{BD}$ & $\mathrm{BD}$ & $\mathrm{BD}$ & $\mathrm{BD}$ & $11.3(5.00-16.1)$ & $5.00(5.00-10.1)$ \\
\hline IFN- $\gamma \mathrm{pg} \cdot \mathrm{mL}^{-1}$ & $1.46(1.40-1.57)$ & $1.68(1.36-2.22)$ & $2.19(2.03-3.59)$ & $2.58(1.96-3.19)$ & $23.6(14.2-35.1)$ & $3.78(2.46-16.7)^{*}$ \\
\hline $\mathrm{MCP}-1 \mathrm{ng} \cdot \mathrm{mL}^{-1}$ & 299 (222-341) & 315 (278-620) & $0.19(0.15-0.24)$ & $0.19(0.18-0.30)$ & $7.18(4.51-10.1)$ & $0.95(0.64-6.10)^{*}$ \\
\hline
\end{tabular}

Data are presented as median (interquartile range). Pneumonia was induced by intranasal inoculation with $5 \times 10^{4}$ Streptococcus pneumoniae CFU. KC: keratinocytederived chemokine; MIP-2: macrophage inflammatory protein-2; TNF- $\alpha$ : tumour necrosis factor- $\alpha$; IL: interleukin; IFN- $\gamma$ : interferon- $\gamma$; MCP-1: monocyte chemotactic protein-1; ND: not determined; BD: below detection. $n=8$ per group in all cases per time-point. ${ }^{*}: p<0.05 ;{ }^{* *}: p<0.01 ;{ }^{* * *}: p<0.001$, statistical significance compared with WT using Mann-Whitney U-test. 


\begin{tabular}{|c|c|c|c|c|c|c|}
\hline \multirow[t]{3}{*}{ TABLE 3} & \multicolumn{6}{|c|}{$\begin{array}{l}\text { Plasma cytokine and chemokine levels in wild-type (WT) mice and mice lacking the lectin-like domain of } \\
\text { thrombomodulin }\left(\mathrm{TM}^{\mathrm{LeD} / \mathrm{LeD}}\right) 6,24 \text { and } 48 \mathrm{~h} \text { post-infection with pneumococcal pneumonia }\end{array}$} \\
\hline & \multicolumn{2}{|c|}{$6 \mathrm{~h}$ post-infection } & \multicolumn{2}{|c|}{$24 \mathrm{~h}$ post-infection } & \multicolumn{2}{|c|}{$48 \mathrm{~h}$ post-infection } \\
\hline & WT & $\mathrm{TM}^{\text {LeD/LeD }}$ & WT & $\mathrm{TM}^{\text {LeD/LeD }}$ & WT & $\mathrm{TM}^{\text {LeD/LeD }}$ \\
\hline TNF- $\alpha \mathrm{pg} \cdot \mathrm{mL}^{-1}$ & $5.99(3.28-8.05)$ & $6.47(5.53-7.99)$ & $7.90(6.38-9.75)$ & $1.30(1.30-4.10)^{\star *}$ & $23.3(18.1-37.4)$ & $3.50(1.95-8.70)^{\star *}$ \\
\hline
\end{tabular}

Escherichia coli and Klebsiella pneumoniae, enhancing their phagocytosis by macrophages [24]. By interacting with the Lewis $\mathrm{Y}$ antigen, the lectin-like domain of TM may interfere with lipopolysaccharide binding to CD14, its cognate receptor, thereby dampening downstream pro-inflammatory signal pathways and the release of pro-inflammatory cytokines, and inducible nitric oxide synthase expression. Indeed, a recombinant soluble form of the lectin-like domain of TM reduced Klebsiella-induced inflammatory responses and lethality [24]. Without invoking other mechanisms, this beneficial response would not necessarily be expected in our Gram-positive pneumonia model, as endotoxin is not involved. The clinical relevance of this finding is important to recognise, as soluble thrombomodulin is being studied for use in the clinic [25] and identifying the appropriate application is critical to ensure

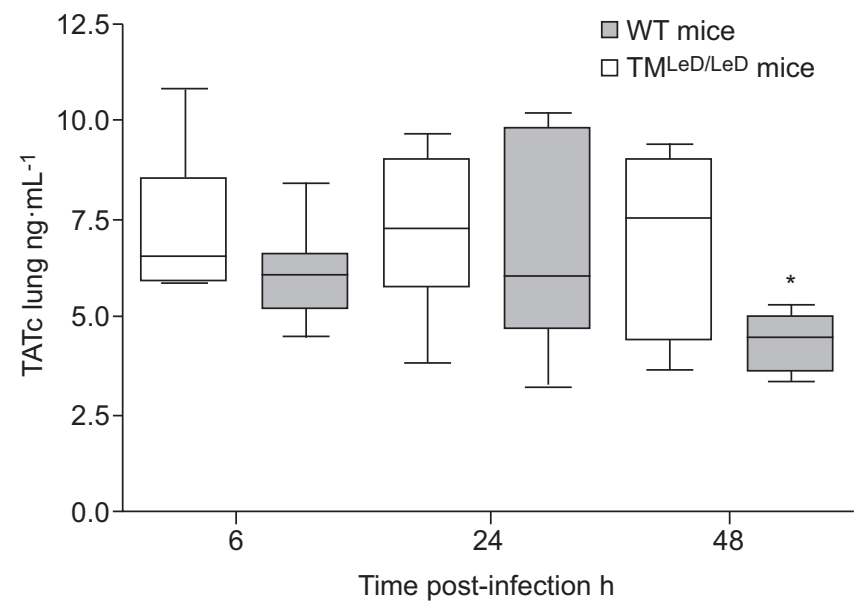

FIGURE 5. Activation of coagulation is reduced in the lung tissue of mice lacking the lectin-like domain of thrombomodulin $\left(\mathrm{TM}^{\mathrm{LeD} / \mathrm{LeD}}\right)$ as compared with wild-type (WT) mice after pneumococcal pneumonia. Lung homogenate levels of thrombin-antithrombin complexes (TATC) 6, 24 and $48 \mathrm{~h}$ post-infection with $5 \times 10^{4}$ Streptococcus pneumoniae CFU in WT and TM $\mathrm{M}^{\mathrm{LeD} / \mathrm{LeD}}$ mice. Data are expressed as box-and-whisker plots depicting the smallest observation, lower quartile, median, upper quartile and largest observation (eight mice per group at each time-point). ${ }^{*}: p<0.05$, statistical significance as compared with WT using Mann-Whitney U-test. optimal benefit. Also, whereas in nonbacterial stress models, anti-inflammatory responses tend to ameliorate damage, in live bacterial models, a substantial amount of inflammatory response is necessary to kill the bacteria; interfering with bacterial killing by reducing the inflammatory response can enhance bacterial outgrowth, in turn resulting in an adverse outcome.

Our study has limitations. As elaborated above, we studied a single pathogen in a single infection model. It is unclear if results from our study would be comparable to those in other models and/or using other bacteria. Also, it is possible that genetic knock-down of $\mathrm{TM}^{\mathrm{LeD} / \mathrm{LeD}}$ has induced unknown compensatory changes in mice during growth and development. In this regard, it would be interesting to study the effect of interfering with the function of the $\mathrm{TM} \mathrm{MeD}^{\mathrm{LeD}}$ domain as opposed to knocking down $\mathrm{TM}^{\mathrm{LeD} / \mathrm{LeD}}$, for example, by using blocking antibodies.

In conclusion, we show that deletion of the lectin-like domain of TM in mice results in improved host defence against pneumococcal pneumonia, a common cause of serious communityacquired infection. Additional studies are necessary to obtain further insight into the possible differential role of the lectin-like TM domain in Gram-positive versus Gram-negative infections, as this will determine how to best design targeted therapies to either enhance the function or interfere with the function of this interesting endothelial C-type lectin-like structure, thereby gaining maximal benefit in different clinical situations.

\section{SUPPORT STATEMENT}

M. Schouten is supported by a research grant of the Dutch Thrombosis Foundation (grant number TSN 2005-1). J.D. de Boer is supported by a grant from the Netherlands Asthma Foundation (project number 3.2.08.009)

\section{STATEMENT OF INTEREST}

None declared.

\section{ACKNOWLEDGEMENTS}

The authors thank the following people, all working at the Center for Experimental and Molecular Medicine, Academic Medical Center, Amsterdam, the Netherlands: M. ten Brink and J. Daalhuisen for their technical assistance during the animal experiments, R. de Beer for performing (immuno)histopathological stainings and A. Achouiti and F.E. van den Boogaard for assisting in cytokine measurements. 


\section{REFERENCES}

1 Mandell LA, Wunderink RG, Anzueto A, et al. Infectious Diseases Society of America/American Thoracic Society consensus guidelines on the management of community-acquired pneumonia in adults. Clin Infect Dis 2007; 44: Suppl. 2, S27-S72.

2 Centers for Disease Control and Prevention. Epidemiology and Prevention of Vaccine-Preventable Diseases. Atkinson W, Wolfe S, Hamborsky J, eds. 12th Edn. Washington DC, public Health Foundation, 2012.

3 Balakrishnan I, Crook P, Morris R, et al. Early predictors of mortality in pneumococcal bacteraemia. J Infect 2000; 40: 256-261.

4 Bartlett JG, Dowell SF, Mandell LA, et al. Practice guidelines for the management of community-acquired pneumonia in adults. Clin Infect Dis 2000; 31: 347-382.

5 Conway EM. Thrombomodulin and its role in inflammation. Semin Immunopathol 2012; 34: 107-125.

6 Ito T, Maruyama I. Thrombomodulin: protectorate God of the vasculature in thrombosis and inflammation. I Thromb Haemost 2011; 9: Suppl. 1, 68-73.

7 Takagi T, Taguchi O, Toda M, et al. Inhibition of allergic bronchial asthma by thrombomodulin is mediated by dendritic cells. Am J Respir Crit Care Med 2011; 183: 31-42.

8 Schouten M, Wiersinga WJ, Levi M, et al. Inflammation, endothelium, and coagulation in sepsis. J Leukoc Biol 2008; 83: 536-545.

9 Danese S, Vetrano S, Zhang L, et al. The protein C pathway in tissue inflammation and injury: pathogenic role and therapeutic implications. Blood 2010; 115: 1121-1130.

10 Weiler H. Regulation of inflammation by the protein C system. Crit Care Med 2010; 38: Suppl. 2, S18-S25.

11 Bajzar L, Manuel R, Nesheim ME. Purification and characterization of TAFI, a thrombin-activable fibrinolysis inhibitor. $J$ Biol Chem 1995; 270: 14477-14484.

12 Myles T, Nishimura T, Yun TH, et al. Thrombin activatable fibrinolysis inhibitor, a potential regulator of vascular inflammation. J Biol Chem 2003; 278: 51059-51067.

13 Conway EM, Van de Wouwer M, Pollefeyt S, et al. The lectin-like domain of thrombomodulin confers protection from neutrophilmediated tissue damage by suppressing adhesion molecule expression via nuclear factor $\kappa \mathrm{B}$ and mitogen-activated protein kinase pathways. J Exp Med 2002; 196: 565-577.
14 Geudens N, Van de Wouwer M, Vanaudenaerde BM, et al. The lectin-like domain of thrombomodulin protects against ischaemiareperfusion lung injury. Eur Respir J 2008; 32: 862-870.

15 Van de Wouwer M, Plaisance S, De Vriese A, et al. The lectin-like domain of thrombomodulin interferes with complement activation and protects against arthritis. J Thromb Haemost 2006; 4: 1813-1824.

16 Kawanami O, Jin E, Ghazizadeh M, et al. Heterogenous distribution of thrombomodulin and von Willebrand factor in endothelial cells in the human pulmonary microvessels. J Nihon Med Sch 2000; 67: 118-125.

17 Ford VA, Stringer C, Kennel SJ. Thrombomodulin is preferentially expressed in Balb/c lung microvessels. J Biol Chem 1992; 267: 5446-5450.

18 Rijneveld AW, Weijer S, Florquin S, et al. Thrombomodulin mutant mice with a strongly reduced capacity to generate activated protein $C$ have an unaltered pulmonary immune response to respiratory pathogens and lipopolysaccharide. Blood 2004; 103: 1702-1709.

19 Schouten M, van't Veer C, Roelofs JJ, et al. Impact of the factor V Leiden mutation on the outcome of pneumococcal pneumonia: a controlled laboratory study. Crit Care 2010; 14: R145.

20 Rijneveld AW, Levi M, Florquin S, et al. Urokinase receptor is necessary for adequate host defense against pneumococcal pneumonia. J Immunol 2002; 168: 3507-3511.

21 Sun K, Metzger DW. Inhibition of pulmonary antibacterial defense by interferon-gamma during recovery from influenza infection. Nat Med 2008; 14: 558-564.

22 Nishimura T, Myles T, Piliponsky AM, et al. Thrombin-activatable procarboxypeptidase $\mathrm{B}$ regulates activated complement $\mathrm{C} 5 \mathrm{a}$ in vivo. Blood 2007; 109: 1992-1997.

23 Brown JS, Hussell T, Gilliland SM, et al. The classical pathway is the dominant complement pathway required for innate immunity to Streptococcus pneumoniae infection in mice. Proc Natl Acad Sci USA 2002; 99: 16969-16974.

24 Shi CS, Shi GY, Hsiao SM, et al. Lectin-like domain of thrombomodulin binds to its specific ligand Lewis $\mathrm{Y}$ antigen and neutralizes lipopolysaccharide-induced inflammatory response. Blood 2008; 112: 3661-3670.

25 Saito H, Maruyama I, Shimazaki S, et al. Efficacy and safety of recombinant human soluble thrombomodulin (ART-123) in disseminated intravascular coagulation: results of a phase III, randomized, double-blind clinical trial. J Thromb Haemost 2007; 5: 31-41. 